\title{
Discrimination Between Fantastic and Ordinary Visual Displays by Children and Adults
}

\author{
Eugene Subbotsky*
}

Psychology Department, Lancaster University, UK

\begin{abstract}
Two experiments tested the ability to distinguish between ordinary and fantastic complex visual displays in 6and 9-year-old children and adults. In Experiment 1, adults found discrimination between ordinary and fantastic visual realities (Task 1) as clear and manageable as the discrimination between pictures that included or did not include human characters (Task 2), but 6- and 9-year-old children did significantly better on Task 2 than on Task 1. Children performed significantly poorer than adults on discrimination between ordinary and fantastic pictures. Other data confirmed that this difference in accuracy of discrimination between 9-year-olds and adults can't be explained by the 9-year-olds' general cognitive deficit. This suggests that children's understanding of the difference between pictures representing ordinary and fantastic realities is a result of special experience with magical reality, rather than a result of general cognitive growth. On both tasks, six-year-olds performed significantly worse than 9-year olds. This supports the hypothesis that there is a developmental progression on the capacity to discriminate between ordinary and fantastic visual realities. In Experiment 2, the same tasks were given to adult participants without identifying the criterion for classification. For Task 1, participants spontaneously used the ordinary/fantastic distinction as a criterion for classification with the frequency significantly above chance, but for Task 2, the frequency of the in-built criterion (presence or absence of people) dropped down to chance level. The results show salience of the distinction between ordinary and fantastic visual realities.
\end{abstract}

Keywords: fantastic reality, ordinary reality, magical thinking, discrimination, classification.

\section{INTRODUCTION}

This study tested children's and adults' ability to discriminate between ordinary and fantastic $(\mathrm{O} / \mathrm{F})$ types of complex visual displays. For ordinary visual reality, visual displays depict ordinary events, even if these events are framed in an extravagant artistic style. In contrast, fantastic visual reality implies pictures of events or objects that violate known physical principles or features of commonly known objects (like flying people or creatures that combine features of people and animals).

The capacity of discriminating between $\mathrm{O} / \mathrm{F}$ types of reality is fundamental for maintaining the normal state of mind. When watching a movie, a person has to constantly judge whether the scene he or she is watching depicts ordinary or fantastic events. Depending on this judgment, the person will treat the movie as a realistic or fantastic reflection of real life. Similarly, when involved in a conversation with other people, we need to be aware of whether or not the partner with whom we are communicating keeps his or her speech within the rules expected from a person referring to the ordinary reality. If these rules are violated, we have to decide if the topic of conversation, whether due to normal (a joke in conversation or a metaphor used to express certain emotional states) or abnormal (speaking with a mentally unstable person) circumstances,

*Address correspondence to this author at the Psychology Department, Lancaster University, UK; Tel: +1524593832; Fax: +01524593744;

E-mail: e.subbotsky@lancaster.ac.uk has shifted from ordinary into fantastic reality. Even within our own mental world, we have to pay constant attention to whether our thoughts or images are within the boundaries of ordinary reality or if they have shifted into the area of fantasy or magical thinking ${ }^{1}$.

The ability to distinguish between ordinary and fantastic realities is linked to the distinction between fantasy and reality. Although not every kind of fantasy involves fantastic reality ${ }^{2}$, all fantastic reality is fantasy. Distinguishing fantasy from reality is important because it mediates the effect of mass media on children's and adults' subsequent behavior. It has been shown, for instance, that children who were aware that a violent film clip was real later reacted more aggressively than children who believed that the film was a fantasy [2]. With children's growing ability to distinguish between fiction and reality, the children's exposure to TV violence may have less impact [3]. Similar mediating effect the fantasy-reality distinction ability may have on children's vulnerability to violent video and computer games [4]. On this ground, it can be assumed that

\footnotetext{
${ }^{1}$ By magical thinking the kind of thinking is understood, which comprises supernatural events or operations (flying on a broomstick, a person turning into an animal, animals speaking human languages, etc.). The key feature of magical thinking is the ability to construct a world that is alternative to the real world. Magical thinking is unfolding whenever a person is involved in some kind of mental processing of supernatural events (i.e., through seeing magical events in a dream, reading a book, or watching a movie with magical content). It has been shown that involvement in magical thinking facilitates creativity in children [1].

${ }^{2}$ For example, much of TV's production is fantasy (i.e., is based on fiction), yet it does not include violation of fundamental physical laws.
} 
children's growing ability to distinguish between ordinary and fantastic realities may produce similar results: children who are better at distinguishing fantastic reality from ordinary one may be less vulnerable to those media products (such as video and computer games) that include elements of fantastic reality (i.e., scenes that violate fundamental physical principles).

The number of studies on the distinction between $\mathrm{O} / \mathrm{F}$ realities is limited. In developmental research, 3- to 5-yearold children found it difficult to distinguish between pictures of real and fantastical animals [5], and this capacity increased, albeit slowly, by the age of eleven [6]. Children aged 4, 7 and 10 years, just like adults, attributed "real" or "unreal" statuses to "monsters" featured in the pictures irrespective of the degree to which these monsters were regarded by them as fearsome or harmless [7]. Sharon and Woolley [8] employed a new measure - a property attribution task. They found that 4- and 5-year-olds' abilities to differentiate between properties of real (child, clown) and fantastic (Santa, Fairy, Superman) entities were the same as in adults, although the children's capacity to correctly categorize the fantastical entities was much inferior to the one of adults.

For healthy adults, categorization between fantastic and real characters doesn't seem to be a problem [7, 8]. Studies on adults looked at more specific abilities, such as retention of plausible versus bizarre mental images [9, 10]. Whereas some bizarre sentences used in these studies might indeed reflect the difference between $\mathrm{O} / \mathrm{F}$ realities (the fish living in the pond versus the fish speaking on the telephone), most of the bizarre images used in these studies (like a clock with a wine bottle used as an arrow or a doctor sitting in the bowl) were not really fantastic [11]. As a result, these studies targeted the distinctiveness of the memory effect on bizarre and regular images rather than on $\mathrm{O} / \mathrm{F}$ realities. Some studies targeted disturbing the ability to discriminate between $\mathrm{O} / \mathrm{F}$ realities in schizophrenic patients, particularly regarding their beliefs in magical causality. Schizophrenic patients tended to engage in magical thinking to a considerably larger extent than control subjects [12]. Healthy participants who answered questionnaires in a similar way to schizophrenic patients also showed a stronger credulity toward magical events then control participants [13]. A stronger tendency to believe in magical events was found in schizophrenics if compared with non-schizophrenic psychiatric patients and control subjects [14]. Schizophrenic patients also tended to endow fantasy items with qualities of objectivity and existence [15] and showed a stronger belief in the reality of paranormal events then did control participants [16].

Finally, one more line of studies targeted the ways people in different cultures draw the distinction between $\mathrm{O} / \mathrm{F}$ realities. These studies showed dramatic differences between Western and non-Western individuals in attributing imagery and hallucinations with reality [17-20].

One key feature of a classification criterion is its salience. In picture classification research, salience of a classificatory cue is the measure of the cue's capacity for discrimination, with the cue providing the highest discrimination rate being the most salient [21]. Another concept that is relevant to this study is the distinction between supervised and unsupervised classification.
Supervised classification is discrimination between classes in which the classes are defined by the system designer, whereas unsupervised classification (clustering) is classification in which the classes are independently determined by a subject based on the similarity of patterns [22]. It can be safely assumed that of the two or more competing classification criteria, the criterion that is most salient produces the largest number of discriminations under the condition of unsupervised classification.

One hypothesis tested in the current study was that there is a developmental progression in humans' ability to understand the distinction between fantastic and ordinary visual displays. While the earlier research showed that preschool [8] and elementary school [6, 7] children experience a difficulty at categorizing between realistic and fantastic entities, it remains unclear whether this difficulty is a result of children's general cognitive deficits (such as in attention or information processing), or a result of children's lack of understanding that fantastic figures include features that violate known physical principles. For example, Sharon \& Wooley [8] showed that if the task on the distinction between fantastic and real entities distinction was made cognitively more appropriate to young children (as the property attribution task was), children's performance on this task was close to that in adults. This suggests that much of children's inability to discriminate between fantastic and realistic entities in categorization tasks could be explained by their general cognitive deficit, rather than by the lack of understanding of the boundary between ordinary and fantastic realities. In this study, the above problem is assessed by comparing between children's and adults' performances on two categorization tasks which are similar in cognitive complexity but different in the criterion used for categorization: one of these tasks employed the ordinary versus fantastic reality distinction for the categorization criterion, whereas the other one didn't.

If the hypothesis about children's developmental progression in the understanding of the distinction between ordinary and fantastic characters was supported, then the task of finding the age at which children are most vulnerable to the potentially damaging visual displays (such as TV, video and computer games' violence) would become practically important. This would also suggest that, rather than simply waiting for the improvement in children's general cognitive abilities, training children on their ability to distinguish fantastic reality from ordinary one could have a positive effect on their ability to disregard the negative media displays.

The second hypothesis of the current study was that the distinction between $\mathrm{O} / \mathrm{F}$ types of visual reality is salient as a criterion for unsupervised classification of complex visual displays. Indeed, in natural environments the mind has to spontaneously, and sometimes rapidly, determine classification cues for discriminating between visual displays. If the $\mathrm{O} / \mathrm{F}$ reality distinction is vital for maintaining the normal state of mind, then people should be expected to use it as a criterion for discriminating between visual displays more often than other possible criteria. In contrast, distinctions based on particular features and not on realities under similar circumstances will not be preferred as criteria to alternative distinctions. 
The current study tested these hypotheses using the task categorization of visual displays that presented pictures of paintings. The reason for using paintings was that in paintings known physical principles are often suspended, and ordinary features of known objects are distorted. In fact, some trends in art, such as surrealism or cubism, made creating fantastic visual realities their main objective. The advantage of using paintings over other material (i.e., sentences) is that the assessment of paintings targets participants' non-verbal judgments and can, therefore, be widely applied across different age and culture groups of participants independently of their language, health, intellectual level and educational background. This test can also be applied to clinical populations, with the aim of comparing the ability to discriminate between $\mathrm{O} / \mathrm{F}$ realities in participants with mental health problems (like frontal lobes patients) ${ }^{3}$ with that of typically developing individuals.

The perceptual matching task was used to assess people's capacity to discriminate between $\mathrm{O} / \mathrm{F}$ realities because this task proved to be a reliable method of assessing perceptual discrimination between visual displays [24].

Since a new test methodology was applied in this study, the study had to be done in two steps: creating the appropriate tasks via a pilot experiment and then testing the main hypotheses. The aim of the pilot study was therefore to develop two tasks on discrimination between complex visual displays that would be similar in their demands to participants' cognitive skills, yet different in their criteria of classification, with only one task having the ordinary/fantastic distinction for such a criterion.

Pilot study Testing the baseline capacity of discriminating between ordinary and fantastic realities supervised classification.

\section{METHOD}

Participants A total of 65 university undergraduate and graduate students from Lancaster University were recruited through the web based recruitment system, 35 in Condition 1 (fixed order) and 30 in Condition 2 (random order). Mean ages and age ranges were $\mathrm{M}=20.6,19$ to 27 , and $\mathrm{M}=19.9,18$ to 22 , for conditions one and two, respectively.

Participants Since one of the hypotheses of the study was to examine the salience of the $\mathrm{O} / \mathrm{F}$ reality criterion of classification against non-salience of more specialized criteria, this criterion had to be contrasted with a specialized criterion. Out of many specialized criteria on which pictures could be classified, presence of people in the picture (present or absent) was selected for a control test on salience. The reason for this selection was that most other specialized criteria, such as the type of landscape (rural or urban) or the presence of animate characters (present or absent), lack universality: for instance, many paintings include both rural and urban elements, and some paintings depict animated non-animate things (like a fish in the shape of a cigar). In contrast, the "presence of people" feature is universal and easily detectable in every display.

\footnotetext{
${ }^{3}$ Some clinical psychologists link schizophrenia with a deficiency in frontal lobe functioning [23].
}

Both the ordinary versus fantastic reality $(\mathrm{O} / \mathrm{F})$ and people versus no people $(\mathrm{P} / \mathrm{N})$ tests consisted of triplets featuring photographs of paintings or their fragments taken from art books. The experiment was controlled by a G5 Mac laptop computer with a17-inch color monitor, and the program was supported by the PsyScript software package. A picture at the top of the screen was the target picture, and two pictures at the bottom were the choice pictures. One of the bottom pictures belonged to the same class as the target picture, and the other one belonged to the opposite class. The target picture was located on the middle line of the screen, and the choice pictures were located on both sides of this line. For half of the presentations, the correct choice picture was to the right, and for the other half it was to the left of the middle line. The participant's task was to click on the bottom picture that belonged to the same class as the target picture. In order to equalize the visual complexity factor, most pictures of both tests were taken from the same pool of pictures, and the remaining pictures were matched by complexity. In both tests, the distracter feature was that of the color scheme: the right choice picture always had a different color scheme from the target picture (i.e., if the target picture was colored, then the correct choice picture was black and white, and vice versa), and the wrong choice picture was always the same color scheme as that of the target picture. This made it possible to use the color scheme (colored versus black and white) as a criterion for classification alternative to the one identified in the instruction. On top of that, each display also allowed for one or more other criteria for classification: pictures having versus not having living things in them, rural versus urban environment, a single object versus multiple objects, and so on.

\section{Design and Procedure}

A within-subjects variable was Test $(\mathrm{O} / \mathrm{F}$ reality distinction versus $\mathrm{P} / \mathrm{N}$ distinction), and a between-subjects variable was Condition. In Condition 1, the order of the items presented was fixed, and in Condition 2 it was random. The number of correct responses was a dependent variable.

In Condition 1, each test included 5 practice trials and 50 main trials. In Condition 2, the number of main trials in both tests was reduced to 42 by eliminating those items of Condition 1 that produced a chance level performance. In order to equalize the number and cognitive difficulty of trials in both tests, in Condition 2, along with the two ambiguous items, six more items which produced the smallest numbers of errors were removed from the $\mathrm{P} / \mathrm{N}$ test.

Each participant was asked to do both $\mathrm{O} / \mathrm{F}$ and $\mathrm{P} / \mathrm{N}$ tests. The order of the tests' presentations was counterbalanced. The $\mathrm{O} / \mathrm{F}$ test was introduced with the following instruction:

"In this experiment there are two types of pictures: those that can be real and those that cannot. First, look at the target picture at the top of the screen. Out of the two pictures at the bottom of the screen, click on the one you think is the same type as the target picture".

The experimenter then proceeded by displaying five test triplets and discussing them with a participant. The instruction for the main test then followed: "End of test. Now start main trial. Don't say anything to the experimenter, 
Table 1. Mean numbers of Correct Responses (SDs) as a Function of Test (O/F versus P/N) and Condition (1-fixed Order Versus 2Random Order)

\begin{tabular}{|c|c|c|c|c|}
\hline Test & \multicolumn{4}{|c|}{ Condition } \\
\hline & 1 (fixed order) & Above chance & 2 (random order) & Above chance \\
\hline \hline O/F & $43.89(3.45)$ & .001 & $38.97(2.96)$ & .001 \\
\hline P/N & $46.43(3.87)$ & .001 & $39.73(2.06)$ & .001 \\
\hline
\end{tabular}

just click on the picture of your choice below the target picture". Participants were then encouraged to proceed to the end of the test independently. The need for quick responding was not emphasized.

The procedure for the $\mathrm{P} / \mathrm{N}$ test was the same as above except that in the first sentence the instruction said: "In this experiment there are two types of pictures: those that have people in them and those that have not".

After the session ended, all hits and misses and for each participant were displayed and then summarized using an Excel spreadsheet.

\section{RESULTS}

Mean numbers of correct responses (SDs) as a function of Test and Condition are shown in Table 1. In both tests, mean numbers of correct responses were reliably above chance level.

In Condition 1, mean number of correct responses on the $\mathrm{P} / \mathrm{N}$ test was significantly higher than that on the $\mathrm{O} / \mathrm{F}$ test (Wilcoxon $\underline{z}=3.053, \mathrm{~N}$-Ties $=33, \underline{\mathrm{p}}<.01$, two tailed), but in Condition 2 this difference was insignificant. Compared as percentages of the total numbers of test displays, the frequency of correct responses for the $\mathrm{O} / \mathrm{F}$ test in Condition 2 was significantly higher than in Condition 1 (Mann Whitney $\mathrm{U}=254.5, \mathrm{p}<.001)$, but there was no difference in this regard on the $\mathrm{P} / \mathrm{N}$ test.

\section{DISCUSSION}

As the numbers of corrects responses approached 92\%, both tests proved well manageable for participants. Since both tasks involved the same process components (recognition of the type of the target picture, discrimination between the choice pictures, and picking the right picture up), and a general level of structural complexity of pictures in both tests was approximately equal, the better performance on the $\mathrm{P} / \mathrm{N}$ task in Condition 1 could be explained by the higher complexity of certain displays of the $\mathrm{O} / \mathrm{F}$ task if compared with those of the $\mathrm{P} / \mathrm{N}$ task. By eliminating the displays which produced a chance level performance from both tasks, and also a few tasks from the $\mathrm{P} / \mathrm{N}$ test that proved to be too easy to categorize, the aim of the pilot study was achieved: the two categorization tasks were developed that were identical in their demands to participants' cognitive skills, yet different in their categorization criteria.

Indeed, making a decision of whether the reality described in a picture is ordinary or fantastic (the $\mathrm{O} / \mathrm{F}$ task) involves detecting an anomaly in the picture and then deciding whether this anomaly is an artistic exaggeration only or if it involves a violation of laws of ordinary reality.
In contrast, all that the $\mathrm{P} / \mathrm{N}$ task involves is detecting the presence or absence of a single feature -a person. Having developed the above tasks, it was possible to target the first problem of this study - to examine whether children perform poorer than adults on the ordinary/fantastic realities categorization tasks because of their general cognitive deficit or because their understanding of the distinction between ordinary and fantastic visual realities was poorer than that in adults.

\section{MAIN STUDY}

Experiment 1. Comparing the distinction between ordinary and fantastic realities in children and adults supervised classification.

\section{METHOD}

\section{Participants}

Thirty-two typically developing children, males and females, participated in this experiment: 16 children in the younger group (mean age 6.5 , age range 5.5 to 6.11 ) and 16 children in the older group (mean age 9.7, age range 8.6 to 11.4). The younger children attended a preschool centre, and the older children - a primary school in the suburb of Moscow. All the children were Russian native speakers. For the comparison with adults, the data of the pilot experiment, Condition 2, were used.

Apparatus and stimuli Those were the same as in the pilot experiment, Condition 2.

\section{Design and Procedure}

A within-subjects variable was test -- ordinary/fantastic $(\mathrm{O} / \mathrm{F})$ reality distinction versus people/no people $(\mathrm{P} / \mathrm{N})$ distinction, and between variables were age (6 years, 9 years and adults). The number of correct identifications was a dependent variable.

The procedure was like in the pilot experiment, Condition 2.

\section{RESULTS}

Mean numbers of identifications made in accord with the ordinary-fantastic reality and people-no people criteria of discrimination are shown in Table 2.

The table shows that numbers of correct identifications on both tests and in all age groups were above chance. In children, performance on the $\mathrm{O} / \mathrm{F}$ test was significantly worse than on the $\mathrm{P} / \mathrm{N}$ test, Wilcoxon $\mathrm{z}=-2.93, \mathrm{~N}$ - Ties $=15$, $\mathrm{p}=.003$, and $\mathrm{z}=-3.24, \mathrm{~N}$ - Ties $=15, \mathrm{p}=.001$, for 6 - and 9 year-olds, respectively, but in adults this difference between tests was not significant. Between ages, on the O/F test, 6year-olds performed significantly worse then did 9-year- 
Table 2. Mean Numbers of Identifications (SDs) Made in Accord with the Ordinary-Fantastic Reality and People-no People Criteria of Discrimination as a Function of Age (6 Years, 9 Years and Adults), and Test (O/F Versus P/N)

\begin{tabular}{|c|c|c|c|c|}
\hline Age & \multicolumn{3}{|c|}{ Test } & P/N \\
\hline & O/F & Above chance & $33.44(7.50)$ & .001 \\
\hline \hline 6 years & $26.94(6.06)$ & .035 & $39.50(2.70)$ & .001 \\
\hline 9 years & $34.44(6.59)$ & .001 & $39.73(2.06)$ & .001 \\
\hline Adults & $38.97(2.96)$ & .001 & \\
\hline
\end{tabular}

olds, Mann-Whitney $\mathrm{U}=209, \mathrm{p}=.002$, and 9-year-olds did significantly worse than adults, Mann-Whitney $\mathrm{U}=109, \mathrm{p}=$ .002. On the P/N test, 6-year-olds did significantly worse than 9-year-olds, Mann-Whitney $\mathrm{U}=201, \mathrm{p}=.004$, but there was no difference in performance between 9 -year-olds and adults, Mann-Whitney $\mathrm{U}=242, \mathrm{p}=.96$.

\section{DISCUSSION}

As expected, children of both age groups performed significantly worse than adults on the O/F test, and 6-yearolds performed significantly worse than 9-year olds. This supports the hypothesis that there is a developmental progression on the capacity to discriminate between ordinary and fantastic visual realities. The question arises of whether this progression is caused by the improvement in general cognitive capacities, such as attention and information processing, or by the improvement in understanding the difference between ordinary and fantastic realities.

When discriminating between ordinary and fantastic realities, both 6- and 9-year-olds were significantly less accurate on this discrimination than adults. This difference in accuracy of discrimination between 9-year-olds and adults cannot be explained by the 9-year-olds' general cognitive deficit. Indeed, on the P/N task, which placed the same cognitive demands on participants' performance as did the $\mathrm{O} / \mathrm{F}$ task and required the same level of attention, 9-year-olds performed as well as adults. This suggests that 9-year-olds' understanding of the difference between pictures representing ordinary and fantastic realities was poorer than that in adults.

In regard to 6-year olds, their poor performance on the $\mathrm{O} / \mathrm{F}$ task could be explained by their poor understanding of the difference between ordinary and fantastic realities as well. However, this poor performance could also be aggravated by cognitive deficits. Indeed, 6-year-olds showed a significantly lower success rate on the discrimination between pictures with and without people than showed either 9 -year-olds or adults, thus suggesting that their general level of cognitive abilities was not as good as in other categories of participants.

Removing the ambiguous items in Condition 2 of the pilot experiment resulted in the disappearance of significant differences between numbers of correct responses on $\mathrm{O} / \mathrm{F}$ and $\mathrm{P} / \mathrm{N}$ tests in adults. This showed that, unlike children, adults were able to perform on both types of discrimination equally well when the criteria for discrimination were made clear to them. This made testing the salience hypothesis possible. Specifically, would adult participants use the main criterion coded into the structure of all items (i.e., ordinary versus fantastic reality and people versus no-people in the $\mathrm{O} / \mathrm{F}$ and $\mathrm{P} / \mathrm{N}$ tests, respectively) in the condition of unsupervised classification? In other words, would participants spontaneously prefer to classify the $\mathrm{O} / \mathrm{F}$ test on the basis of an ordinary-fantastic reality distinction if the opportunity of using this criterion were not mentioned in the instruction?

In order to examine this, Experiment 2 was conducted.

Experiment 2. Testing salience of the distinction between ordinary and fantastic realities - unsupervised classification.

\section{METHOD}

Participants. Thirty-two university undergraduate and graduate students, males and females, participated in this experiment. Mean ages and age ranges were $\mathrm{M}=22.3,18$ to 28 .

Apparatus and stimuli These were the same as those in Experiment 1.

\section{Design And Procedure}

A within-subjects variable was Test $(\mathrm{O} / \mathrm{F}$ versus $\mathrm{P} / \mathrm{N})$, and a mixed between-within-subjects variable was Type of classification - supervised versus unsupervised. The number of correct responses was a dependent variable.

Each participant was asked to do both the $\mathrm{O} / \mathrm{F}$ and $\mathrm{P} / \mathrm{N}$ tests, one with the directive instruction (like in Experiment 1) and the other with the open instruction. The open instruction was as follows: "In this experiment there are two types of pictures. First, look at the target picture at the top of the screen. Out of the two pictures at the bottom of the screen, click on the one you think is the same type as the target picture..." The rest of the instruction was like in Experiment 1 and identical with the directive instruction.

For half of the participants, the instruction in the $\mathrm{O} / \mathrm{F}$ test was open, and in the $\mathrm{P} / \mathrm{N}$ test it was directive, and for the other half the order was reversed. The order of the $\mathrm{O} / \mathrm{F}$ and $\mathrm{P} / \mathrm{N}$ tests presentation was counterbalanced across participants.

\section{RESULTS}

Mean numbers of classifications made in accord with the $\mathrm{O} / \mathrm{F}$ and $\mathrm{P} / \mathrm{N}$ criteria of discrimination are shown in Table 3 and illustrated in Fig. (1).

The table shows that numbers of correct responses in the supervised condition (directive instruction) on both tests were high above chance and approached $92 \%$ of the total number of items. In the unsupervised condition (open instruction), the number of responses made on the basis of 
Table 3. Mean numbers of identifications (SDs) made in accord with the ordinary-fantastic reality and people-no people criteria of discrimination as a function of Test $(\mathrm{O} / \mathrm{F}$ versus $\mathrm{P} / \mathrm{N})$ and Type of classification (supervised versus unsupervised).

\begin{tabular}{|c|c|c|c|c|}
\hline Type of Classification & \multicolumn{3}{|c|}{ Test } & P/N \\
\hline & O/F & Above Chance & $39.00(2.75)$ & Above Chance \\
\hline \hline Supervised & $38.25(3.60)$ & .001 & $23.06(5.51)$ & .001 \\
\hline Unsupervised & $28.00(7.99)$ & .007 & .154 \\
\hline
\end{tabular}

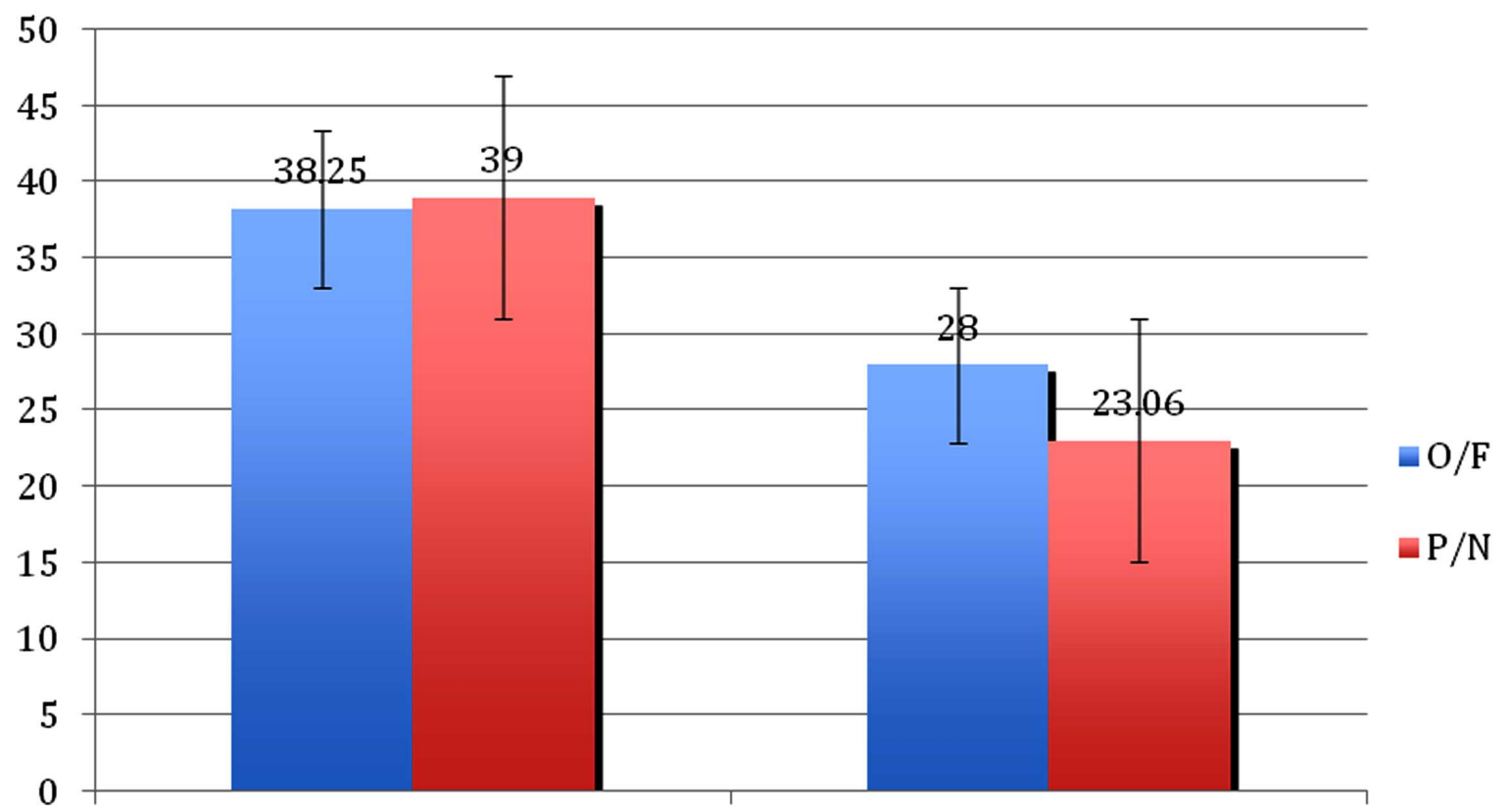

Supervised

Unsupervised

Fig. (1). Mean numbers of identifications (SDs) made in accord with the ordinary-fantastic reality and people-no people criteria of discrimination as a function of Test $(\mathrm{O} / \mathrm{F}$ versus $\mathrm{P} / \mathrm{N})$ and Type of classification (supervised versus unsupervised).

$\mathrm{P} / \mathrm{N}$ criterion in the $\mathrm{P} / \mathrm{N}$ test dropped down to chance (Wilcoxon $\mathrm{z}=1.425, \mathrm{~N}$-Ties $=15, \mathrm{p}=.154,2$-tailed), however, the number of responses made on the basis of $\mathrm{O} / \mathrm{F}$ criterion in the $\mathrm{O} / \mathrm{F}$ test was still significantly above chance (Wilcoxon $\mathrm{z}=-2.674$, N-Ties $=16, \mathrm{p}=.007,2$-tailed).

In the supervised condition, the difference between numbers of correct responses in the $\mathrm{O} / \mathrm{F}$ and $\mathrm{P} / \mathrm{N}$ tests was insignificant (Mann-Whitney $\mathrm{U}=147, \mathrm{~N} 1=\mathrm{N} 2=16, \mathrm{p}=.46$, 2-tailed). In the unsupervised condition, the mean number of identifications based on the $\mathrm{O} / \mathrm{F}$ criterion in the $\mathrm{O} / \mathrm{F}$ test was significantly higher than the mean number of identifications based on the $\mathrm{P} / \mathrm{N}$ criterion in the $\mathrm{P} / \mathrm{N}$ test (Mann-Whitney $\mathrm{U}=$ 74.00, $\mathrm{N} 1=\mathrm{N} 2=16, \mathrm{p}=.043,2$-tailed).

For the participants who did the $\mathrm{O} / \mathrm{F}$ test under the unsupervised condition and the $\mathrm{P} / \mathrm{N}$ test under the supervised condition, the mean number of correct identifications in the $\mathrm{P} / \mathrm{N}$ test was significantly larger than the mean number of identifications on the basis of $\mathrm{O} / \mathrm{F}$ criterion in the $\mathrm{O} / \mathrm{F}$ test (Wilcoxon $\mathrm{Z}=-3.260, \mathrm{~N}$-Ties $=16, \mathrm{p}=.001,2$-tailed), and in participants who did the $\mathrm{O} / \mathrm{F}$ test under the supervised condition and the $\mathrm{P} / \mathrm{N}$ test under the unsupervised condition, the mean number of correct identifications on the $\mathrm{O} / \mathrm{F}$ test significantly exceeded the mean number of identifications made on the basis of $\mathrm{P} / \mathrm{N}$ criterion in the $\mathrm{P} / \mathrm{N}$ test (Wilcoxon $\mathrm{Z}=-3.441, \mathrm{~N}$-Ties $=16, \mathrm{p}=.001,2$-tailed).

\section{DISCUSSION}

The results indicated that under the supervised condition in Experiment 2, the numbers of correct identifications in the $\mathrm{O} / \mathrm{F}$ and $\mathrm{P} / \mathrm{N}$ tests were about the same as in Experiment 1 and close to $92 \%$ of the total number of trials. This indicated that in terms of clarity of distinction both tests were easily manageable. In the unsupervised condition, the number of responses based on $\mathrm{P} / \mathrm{N}$ criterion in the $\mathrm{P} / \mathrm{N}$ test dropped down to chance, whereas in the $\mathrm{O} / \mathrm{F}$ test the number of responses on the basis of $\mathrm{O} / \mathrm{F}$ criterion was still significantly above chance. This suggests that the answer to the main question of this experiment - would participants still prefer the in-built criterion for classification when this criterion were not identified in the instruction - is "yes' in regard to the $\mathrm{O} / \mathrm{F}$ test but "no" in regard to the $\mathrm{P} / \mathrm{N}$ test. In other words, the $\mathrm{O} / \mathrm{F}$ criterion was salient: it was used with a frequency significantly above chance even when the opportunity of using other criteria was open. In contrast, the $\mathrm{P} / \mathrm{N}$ criterion was not salient and only used at chance level.

\section{GENERAL DISCUSSION}

The study confirmed the expectation, based on earlier research, about the developmental progress in children's capacity to distinguish between ordinary and fantastic realities. Under the supervised classification, both 9- and 6- 
year-old children performed on the $\mathrm{O} / \mathrm{F}$ realities task at the level significantly better then chance. At the same time, it is clear that for children discriminating between ordinary and fantastic realities was significantly more difficult than for adults. This supports the earlier reported data that categorization between real and fantastic entities for preschool and elementary school children is difficult $[5,6]$ and a lot more difficult than for adults [8]. The new result that this study added was that 9-year-old children's poor performance on the fantasy/reality categorization (if compared with that of adults) is likely to be the result of poorer understanding of the distinction between ordinary and fantastic realities rather than the result of the deficit in general cognitive processes, such as attention and information processing. The cognitive deficits, however, may have aggravated the performance on the $\mathrm{O} / \mathrm{F}$ realities task in 6-year-olds.

The results of this study also supported the expectation about the salience of the distinction between fantasy and reality. Under the unsupervised classification, adult participants still tended to classify pictures in the $\mathrm{O} / \mathrm{F}$ test on the basis of $\mathrm{O} / \mathrm{F}$ criterion with a frequency above chance (Experiment 2).

How can salience of the $\mathrm{O} / \mathrm{F}$ distinction be explained in terms of the earlier reported data on picture classification? One possible explanation comes from using contentdependent and content-independent criteria in picture classification. In the classification of pictures (for instance, into indoor versus outdoor classes of photographs), contentindependent meta-criteria for classification (such as exposure time) outperform content-dependent low-level cues (such as cues based on color or texture of objects) [21]. In the tasks used in this study, the ordinary/fantastic reality cue can be viewed as a content-independent criterion. Indeed, the defining feature of the ordinary/fantastic reality distinction is a violation of the laws of ordinary reality, and this feature is invariant to the content: a flying person or a flying mountain are equally impossible in real life, independently of the differences between the displays' contents (i.e., in color, types of objects, object's shape or number, etc.). In contrast, specific criteria (such as presence or absence of a person) are content dependent. In the supervised classification condition, the advantage of meta-criteria over specific criteria is undetectable, but it becomes evident under the more difficult unsupervised classification.

The challenging task that remains is to find out at what age children, like adults, start treating the ordinary/fantastic reality distinction as salient. Another task for further research is to establish whether patients who are prone to hallucinatory phenomena, such as patients with schizophrenia, also have problems with the correct discrimination between ordinary and fantastic realities. In the recent decades, studies have shown that patients with schizophrenia perform worse than healthy adults on tasks that depend on contour integration (a subject's ability to link representations of separate visual stimuli into a coherent percept) [25-27]. Patients with schizophrenia have also shown abnormalities in tasks on facial processing and recognition [28-30]. In particular, performance deficits have been observed in this disorder when participants were asked to identify degraded pictures of faces. If, due to these perceptual deficits, schizophrenic patients confuse fantastical and realistic visual displays, this could be viewed as one of cognitive contributors towards the tendency to hallucinations: the confusion between fantasy and reality in real life.

\section{CONFLICT OF INTEREST}

The authors confirm that this article content has no conflicts of interest.

\section{ACKNOWLEDGEMENTS}

Requests for reprints should be sent to Eugene Subbotsky, Psychology Department, Lancaster University, LA1 4YF, UK.

\section{REFERENCES}

[1] Subbotsky E, Hysted C, Jones N. Watching films with magical content facilitates creativity in children. Percept Mot Skills 2010; 111: 261-7.

[2] Atkin C. Effects of realistic TV violence vs. fictional violence on aggression. Journal Quart 1983; 60: 615-21.

[3] Bushman D, Huesmann LR. Effects of televised violence on aggression. In: Singer D, Singer J, Eds. Handbook of children and the media. Thousand Oaks, CA USA: Sage 2001; pp. 223-54.

[4] Comstock G, Scharrer E. Media pop culture. In: Damon W, Lerner RM, Eds. Handbook of Child Psychology. $6^{\text {th }}$ ed. Wiley: New York 2006; vol. 4,

[5] Taylor B, Howell R. The ability of three-, four-, and five-year-old children to distinguish ordinary from fantastic reality. J Gen Psychol 1973; 122: 315-8.

[6] Morrison P, Gardner H. Dragons and dinosaurs: The child's capacity to differentiate ordinary from fantastic reality. Child Dev 1978; 49: 642-8.

[7] Prawat RS, Anderson AH, Hapkiewitcz W. Is the scariest monster also the least real? An examination of children's reality classifications. J Gen Psychol 1983;146: 7-12.

[8] Sharon T, Woolley JD. Do monsters dream? Young children's understanding of the fantasy/reality distinction. Br J Dev Psychol 2004; 22: 293-310.

[9] Cox SD, Wollen KA. Bizarredness and recall. Bull Psychonom Soc 1981; 18: 244-5.

[10] Einstein GO, McDaniel MA, Lackey S. Bizarre imagery, interference, and distinctiveness. J Exp Psychol Learn Mem Cogn 1989; 15: 137-46.

[11] O'Brien EJ, Wolford CR. Effect of delay in testing on retention of plausible versus bizarre mental images J Exp Psychol Learn Mem Cogn 1982; 8: 148-52.

[12] Tissot R, Burnand Y. Aspects of cognitive activity in schizophrenia. Psychol Med 1980; 10: 657-63.

[13] Eckblad M, Chapman LJ. Magical ideation as an indicator of schizotypy. J Consult Clin Psychol 1983; 51: 215-25.

[14] George L, Neufeld RW. Magical ideation and schizophrenia. Special Issue: Eating disorders. J Consult Clin Psychol 1987; 55: 778-9.

[15] Aggernaes A. Reality testing in schizophrenia. Nord J Psychiatry 1994; 48: 47-54.

[16] Thalbourne MA. Belief in the paranormal and its relationship to schizophrenia-relevant measures: a confirmatory study. $\mathrm{Br} \mathrm{J}$ Clin Psychol 1994; 33: 78-80.

[17] Al-Issa I. The illusion of reality or the reality of illusion: Hallucinations and culture. Brit J Psychiatry 1995; 166: 368-73.

[18] Jaynes J. The origin of consciousness in the breakdown of the bicameral mind. Boston: Houghton Mifflin 1976.

[19] Lévy-Brühl L. La mentalité primitive. Paris: Alcan 1925.

[20] Tulviste P. The cultural-historical development of verbal thinking. New York: Nova Science 1991.

[21] Boutell M, Luo J. Beyond pixels: Exploring camera metadata for photo classification. 2004. Available from: http://www.rosehulman.edu/ boutell/publications/boutellIUPR.pdf. 
[22] Jain AK, Duin RPW, Mao J. Statistical pattern recognition: a review. IEEE Trans Pattern Anal Mach Intell 2000; 22: 4-37.

[23] Goldberg E. The executive brain. Frontal lobes and the civilised mind. New York: Oxford University Press 2002.

[24] Lamberts K, Brockdorff N, Heit E. Preceptual processes in matching and recognition of complex pictures. J Exp Psychol Hum Percept Perform 2002; 28: 1176-91

[25] Butler PD, Silverstein SM, Dakin SC. Visual perception and its impairment in schizophrenia. Biol Psychiatry 2008; 64: 40-7.

[26] Silverstein SM, Kovács I. Corry R, Valone C. Perceptual organization, the disorganization syndrome, and context processing in chronic schizophrenia. Schizophr Res 2000; 43: 11-20.
[27] Silverstein SM, Berten S, Essex B, Kovács I, Susmaras T, Little DM. An fMRI Examination of Visual Integration in Schizophrenia. J Integr Neurosci 2009; 8: 175-202.

[28] Uhlhaas PJ, Linden DEJ, Singer W, et al. Dysfunctional long-range coordination of neural activity during Gestalt perception in schizophrenia. J Neurosci 2006; 26: 8168.

[29] Uhlhaas PJ, Phillips WA, Mitchell G, Silverstein SM. Perceptual grouping in disorganized schizophrenia. Psychiatr Res 2006; 145: 105-17.

[30] Joshua N, Rossell S. Configural face processing in schizophrenia. Schizophr Res 1962; 112: 99-103.

(C) Eugene Subbotsky; Licensee Bentham Open.

This is an open access article licensed under the terms of the Creative Commons Attribution Non-Commercial License (http://creativecommons.org/licenses/by$\mathrm{nc} / 3.0 /$ ), which permits unrestricted, non-commercial use, distribution and reproduction in any medium, provided the work is properly cited. 